\title{
Tumor-Associated Lymphatic and Venous Vessels in Medullary Thyroid Carcinomas
}

\author{
Tatsuo Tomita \\ Departments of Integrative Biosciences and Pathology, Oregon National Primate Center, Oregon Health and \\ Science University, Portland, USA \\ Email: tomitat@ohsu.edu
}

Received 24 December 2014; accepted 9 April 2015; published 13 April 2015

Copyright (C) 2015 by author and Scientific Research Publishing Inc.

This work is licensed under the Creative Commons Attribution International License (CC BY).

http://creativecommons.org/licenses/by/4.0/

(c) (i) Open Access

\section{Abstract}

Objective: Medullary thyroid carcinomas (MTCs) invade local lymph node through lymphatic vessels and metastasize to distant organs hematogenously and account for a significant mortality. There are possibly increased lymphatic and venous vessels, through which the tumor spreads to lymph nodes and distant organs. Materials and Methods: By immunocytochemical staining for lymphatic and venous vessels, MTC lesions with adjacent normal thyroid and both normal and metastatic lymph nodes were studied for the peritumoral lymphatic and venous vessels, which were morphometrically compared with those of normal thyroid and lymph nodes. Sixteen cases of MTC cases with adjacent thyroid tissues and attached lymph nodes were immunocytochemically stained for lymphatic vessels using lymphatic vessel hyaluronan receptor (LYVE-1) and venous vessels for factor VIII (F-8). The immunostained sections of MTC lesions and metastatic lymph nodes were morphometrically compared for the number and sizes of the vessels with those of normal thyroid tissues and lymph nodes. Results: Significantly increased lymphatic vessels and markedly increased blood vessels were identified in many MTC cases at the peritumoral tissues and metastatic lymph nodes whereas a few lymphatic vessels and no venous vessels were identified in midst of MTCs. The irregular peritumoral lymphatic vessels resembled that of immature lymphatic vessels observed in papillary thyroid carcinomas and increased irregularly, entrapped venous vessels in peritumoral tissues resembled those observed in follicular thyroid carcinomas. Conclusion: The significantly increased lymphatic vessels and markedly increased venous vessels in the peritumoral thyroid tissue support a propensity of MTCs for providing an easy access of tumor cells to both lymphatic spread to the regional lymph nodes and venous spread to distant organs with further tumor spread through metastatic lymph nodes by moderately increased lymphatic and venous vessels.

\section{Keywords}

Blood Vessels, Chromogranin A, Factor 8, Immmunocytochemistry, Lymphatic Vessels, LYVE-1, 


\section{Medullary Thyroid Carcinoma}

\section{Introduction}

Medullary thyroid carcinomas (MTCs) are relatively rare tumors, representing about $3 \%-10 \%$ of all thyroid carcinomas but account for significant mortality with overall prognosis of $60 \%-70 \%$ of 10 year survival rate [1] [2]. MTCs invade regional lymph nodes and spread hematogenously to distant organs, particularly lungs, liver and bones, which are the major cause of death [2]. Machens and Dralle analyzed 715 cases of MTC and found that $10 \%, 8 \%$ and $5 \%$ were diagnosed with lung, liver and bone metastasis, respectively [3]. Recently, there is an increased detection of micro-MTC thanks to the routine measurement of serum calcitonin levels [4]. Sporadic MTCs account for $80 \%$ of the carcinomas whereas $20 \%$ have a familial basis [5] [6]. Both papillary and follicular carcinomas reportedly showed increased lymphatic vessels and venous vessels, respectively: papillary carcinomas revealed increased lymphatic vessels using lymphatic vessel endothelial hyluronan receptor (LYVE-1) immunostaining [7] and follicular variant of papillary carcinomas showed more increased intratumoral lymphatic vessels using D2-40 immunostaining [8] whereas follicular thyroid carcinomas revealed increased venous vessels using CD 31 as a vascular marker [9]. In this study, LYVE-1 was used as a specific lymphatic vessel marker [8]-[12] and factor VIII (F-8) was used as a venous vessel marker [12] [13] in order to identify both periand intratumoral lymphatic and venous vessels in primary MTCs and regional metastatic lymph nodes.

\section{Materials and Methods}

All tissue samples of normal thyroids and MTCs were collected at the University of Kansas Medical Center between 1975 and 2001 during my tenure at the Medical Center as a staff pathologist. Six normal thyroids, 9 sporadic and 7 MTCs from MEN-2A families were included, the majority of which were previously reported on by us [15]. All tissue samples of normal thyroids and MTCs were routinely fixed in buffered formalin and embedded in paraffin. Deparaffinized sections were treated with antigen retrieval procedure using citrate buffer $\mathrm{pH}$ 6.2. All the staining procedure was the same as previously reported including calcitonin [12]-[14] except for LYVE-1 and factor VIII (F-8) immunostaining: goat antihuman LYVE-1 (R \& D System, Minneapolis, MN) was used at 1:100 dilution and F-8 immunostaining was performed using rabbit antihuman F-8 (Dako, Carpenteria, CA) diluted at 1:100 [12] [13]. In all cases, LYVE-1 and F-8 immunostaining was evaluated at the peri- and intratumoral tissues. For evaluating LYVE-1 and F-8 immunostaining, morphometric analysis was performed using a mounted $1 \mathrm{~cm}$ linear scale with $10 \mu \mathrm{m}$ intervals in the 10X eye piece. The length and width of LYVE-1 positive lymphatic vessels and F-8 positive venous vessels were measured in $\mu \mathrm{m}$ at $10 \times 10=100 \times$ magnification as reported previously [16]. Both lymphatic and venous vessels were measured in the ten $100 \times$ fields for peri- and intra-tumor lesions and for non-tumor lesions, the latter was $>1 \mathrm{~cm}$ away from the MTCs. When lymph nodes were included in the specimens (Cases $2,3,4,10,11,12,13$ and 15), both lymphatic and venous vessels were measured for metastatic lymph nodes and tumor-free lymph nodes, respectively (Table 1).

\section{Results}

In five normal thyroid glands included in Cases 2, 3, 4, 10 and 11 (Table 1), there were randomly distributed collapsed slender to small lymphatic vessels in the fine fibrous inter-follicular connective tissues whereas more numerous venous vessels with narrow to round lumen were located in the very fine connective tissue stroma (Figure 1(A) and Figure 1(B)). There were about 30\% more venous vessels than lymphatic vessels by measuring the total length and width (Table 1). In frozen sections, normal thyroid tissues revealed abundant slender lymphatic endothelia, which were immunostained adjacent to the pseudocapsule and within sporadically distributed interfollicular fibrous stroma (Figure 1(C)). Similarly more numerous slender to round venous vessels in the fibrous stroma were strongly immunostaind adjacent to the pseudocapsule whereas arterial endothelia were weakly immuostained for F-8 (Figure 1(D)) as compared to the negative immunostaining of arterial endothelia in the formalin-fixed and paraffin-embedded tissues (Figure 1(B)). Among 16 cases of MTCs, 9 cases were sporadic and 7 cases were from MEN-2A families, in the latter the youngest patient was 14 years old (Table 1). In the MEN-2A cases, all 7 cases were in either 10's or 20's and 30's (Table 1), in all cases MTC lesions were 
Table 1. MTC cases and LYVE-1 and F-8 immunocytochemical staining.

\begin{tabular}{|c|c|c|c|c|c|c|c|c|c|c|c|}
\hline \multirow[b]{3}{*}{ Case } & \multirow[b]{3}{*}{ Age/Sex } & & \multicolumn{4}{|c|}{ Peritumoral Thyroid } & \multicolumn{5}{|c|}{ Lymph Nodes } \\
\hline & & & \multicolumn{2}{|c|}{ LYVE-1 } & \multicolumn{2}{|c|}{$\mathrm{F}-8$} & \multicolumn{2}{|c|}{ LYVE-1 } & \multicolumn{2}{|c|}{$\mathrm{F}-8$} & \\
\hline & & & Length & Width & Length & Width & Length & Width & Length & Width & \\
\hline 1 & $14 / \mathrm{M}$ & MEA-2B & $70 \mu \mathrm{m}$ & $35 \mu \mathrm{m}$ & $1560 \mu \mathrm{m}$ & $930 \mu \mathrm{m}$ & & & & & \\
\hline \multirow[t]{2}{*}{2} & $23 / \mathrm{F}$ & MEA-2B & 1375 & 50 & 630 & 195 & $800 \mu \mathrm{m}$ & $140 \mu \mathrm{m}$ & $1930 \mu \mathrm{m}$ & $170 \mu \mathrm{m}$ & Meta LN \\
\hline & & & & & & & 225 & 30 & 500 & 145 & $\mathrm{LN}$ \\
\hline \multirow[t]{2}{*}{3} & $26 / \mathrm{F}$ & MEA-2B & 170 & 105 & 565 & 285 & & & & & \\
\hline & & Thyroid & 130 & 90 & 450 & 95 & 410 & 55 & 630 & 195 & $\mathrm{LN}$ \\
\hline \multirow[t]{2}{*}{4} & $26 / M$ & MEA-2B & 490 & 95 & 365 & 50 & & & & & \\
\hline & & Thyroid & 100 & 70 & 310 & 140 & 555 & 125 & 435 & 65 & $\mathrm{LN}$ \\
\hline 5 & $28 / F$ & MEA-2B & 410 & 55 & 630 & 195 & & & & & \\
\hline \multirow[t]{2}{*}{6} & $30 / \mathrm{M}$ & SP & 1040 & 380 & 3360 & 225 & & & & & \\
\hline & & Thyroid & 225 & 60 & 415 & 145 & & & & & \\
\hline \multirow[t]{2}{*}{7} & $31 / \mathrm{M}$ & SP & 190 & 90 & 720 & 315 & & & & & \\
\hline & & Thyroid & 200 & 150 & 305 & 85 & & & & & \\
\hline 8 & $36 / F$ & MEA-2B & 1040 & 345 & 565 & 280 & & & & & \\
\hline 9 & $38 / F$ & MEA-2B & 465 & 90 & 435 & 65 & & & & & \\
\hline \multirow[t]{2}{*}{10} & $49 / \mathrm{F}$ & SP & 555 & 115 & 705 & 305 & 175 & 90 & 1465 & 415 & Meta LN \\
\hline & & & & & & & 205 & 90 & 250 & 115 & $\mathrm{LN}$ \\
\hline \multirow[t]{2}{*}{11} & $53 / \mathrm{F}$ & SP & 205 & 40 & 800 & 185 & 405 & 185 & 705 & 305 & Meta LN \\
\hline & & & & & & & 290 & 35 & 355 & 120 & LN \\
\hline 12 & $56 / F$ & SP & & & & & 175 & 90 & 5250 & 155 & Meta LN \\
\hline \multirow[t]{2}{*}{13} & $57 / \mathrm{F}$ & SP & 2625 & 185 & 3125 & 285 & 525 & 190 & 3450 & 1500 & Meta LN \\
\hline & & Thyroid & 450 & 45 & 80 & 45 & & & & & \\
\hline \multirow[t]{2}{*}{14} & $66 / F$ & SP & 225 & 85 & 360 & 185 & & & & & \\
\hline & & Bone & 235 & 165 & 365 & 170 & & & & & \\
\hline \multirow[t]{2}{*}{15} & $68 / F$ & SP & 465 & 90 & 435 & 65 & 420 & 120 & 1955 & 365 & Meta LN \\
\hline & & Thyroid & 385 & 80 & 365 & 50 & & & & & \\
\hline \multirow[t]{5}{*}{16} & $90 / F$ & SP & 640 & 125 & 6840 & 1125 & & & & & \\
\hline & MTC (15) & Mean & $664^{\mathrm{a}}$ & $126^{\mathrm{b}}$ & $1402^{\mathrm{a}}$ & $302^{\mathrm{b}}$ & $417^{\mathrm{c}}$ & $136^{\mathrm{a}}$ & $2459^{b}$ & $485^{b}$ & Meta LN \\
\hline & & SE & 170 & 27 & 458 & 77 & 96 & 35 & 177 & 207 & $(n=6)$ \\
\hline & Thyroid (6) & Mean & 248 & 83 & 321 & 93 & 337 & 67 & 128 & 128 & $\mathrm{LN}$ \\
\hline & & SE & 57 & 15 & 53 & 17 & 65 & 18 & 21 & 21 & $(n=5)$ \\
\hline
\end{tabular}

SP: Sporadic; LN: Lymph Node; Meta LN: Metastatic Lymph Node; MTC cases compared with normal thyroid and metastatic lymph nodes compared with normal lymph nodes: a: $\mathrm{p}<0.001$, b: $\mathrm{p}<0.01$, c: $\mathrm{p}=$ non-significant.

bilateral. The MTC bilateral lesions were multifocal and relatively small $(<1 \mathrm{~cm})$, which were surrounded by in situ MTC lesion or C-cell hyperplasia (figure not shown). Peritumoral lymphatic vessels were slightly to more increased from one area to another (Figure 2(A) and Figure 2(C)). Peritumoral thyroid tissues showed 2.6 time more lymphatic vessels and 4.2 times more venous vessels than the normal thyroid tissues by the total vessel length and contained more than twice venous vessels than lymphatic vessels (Figure 2(A)-Figure 2(D), Table 1). Venous vessels were more roundish with larger length and width at twice as larger than that of lymphatic vessels (Figure 2(A)- 


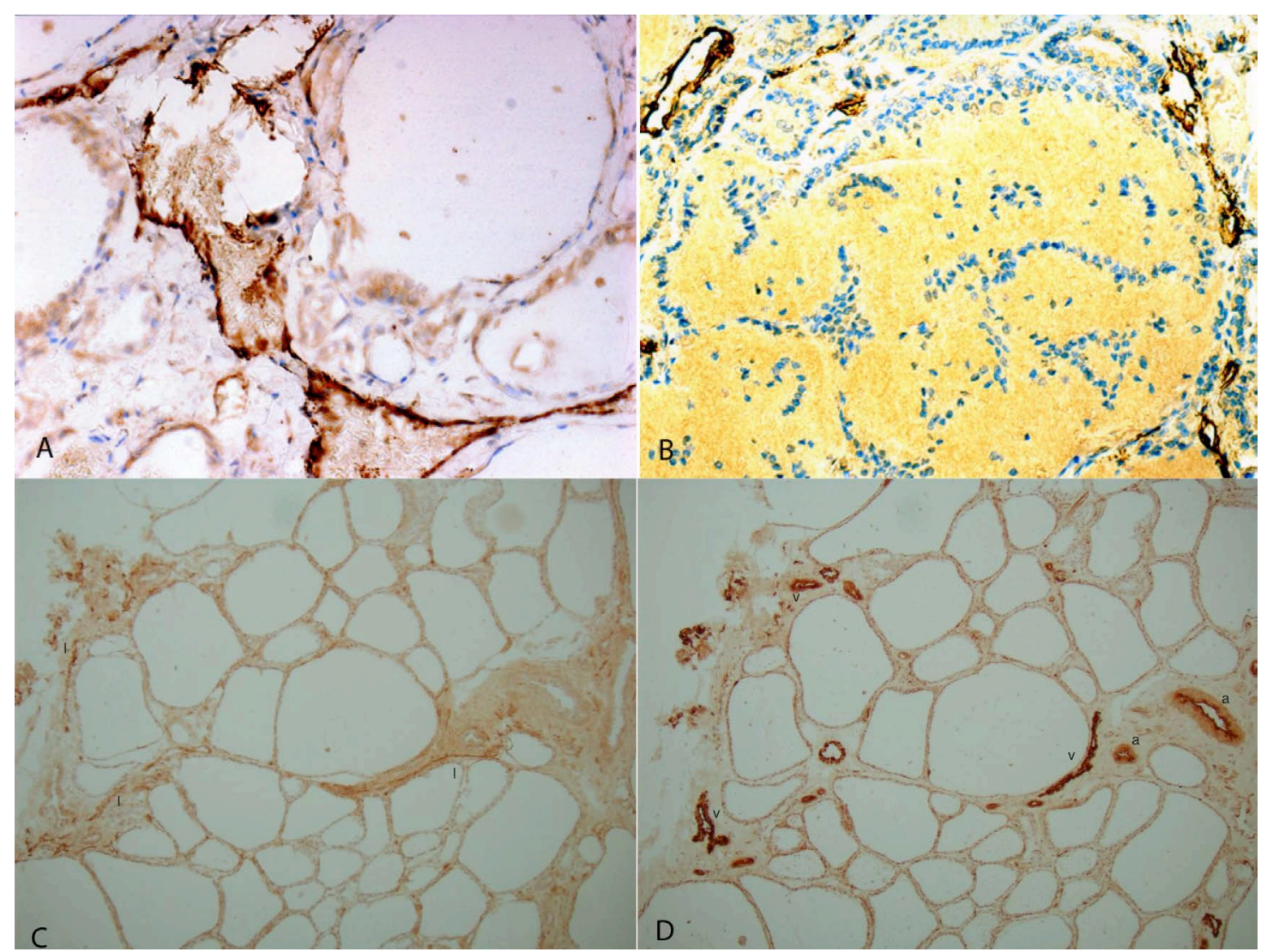

Figure 1. Normal thyroid. There were sporadically located collapsed slender to narrow lymphatic vessels (I) (A) and numerous dilated blood vessels (v) (B) of various sizes in interfollicular stroma in the formalin-fixed and paraffin-embedded sections. More numerous slender venous vessels with narrow to round lumen were in the fine fibrous stroma between thyroid follicles (B). Immunostaining with frozen sections revealed many slender to round lymphatic vessels in the pseudocapsule and larger slender lymphatic vessels (I) in the perivasculal fibrous stroma (C). Round strongly immunostained venous vessels (v) were abundantly present in the pseudocapsule and perivascular fibrous stroma where lesser immunostained endothelia of arteries (a) were also depicted (D); (A) and (C): LYVE-1, (B) and (D): F-8.

Figure 2(D), Table 1). There were relatively few lymphatic and venous vessels in the midst of the tumors despite the occasional slender lymphatic and venous vessels at the peripheral tumor (Figure 2(A) and Figure 2(B)). There were no tumor emboli or lymphovascular invasion in the lymphatic and venous vessels.

Six cases of metastatic and five cases of non-metastatic lymph nodes were available for this study from five sporadic cases (Cases 10, 11, 12, 13, and 15) and three MEN-2 cases (Cases 2, 3 and 4): Normal lymph nodes included in Cases 2, 3, 4, 10 and 11 (Table 1) revealed lymphatic vessels with thin immunopostive endothelia forming narrow to round lumens whereas venous vessels revealed more numerous slender to round lumens (Figure 3(A) and Figure 3(B)). Metastatic lymph nodes in Cases 2, 10, 11, 12, 13 and 15, Table 1) revealed abundant slender to round venous lumens at the peripheral margin of the metastatic tumors continuous to perilymph node connective tissue (Figure 3(E)). Metastatic lymph nodes showed 24\% more lymphatic vessels and 50\% more venous vessels than that of the tumor-free lymph nodes by the total vessel length, the former contained about 5.9 times more venous vessels than lymphatic vessels by the total vessel length with small venous vessels in the midst of metastatic tumor (Figure 3(A)-Figure 3(F), Table 1).

\section{Discussion}

Papillary thyroid carcinomas have a propensity for intrathytroid spread by lymphatic vessels with further lymphagenous metastases to the regional neck lymph nodes [1]. There was a strong association between the intratumoral lymphatic vessels and regional lymph node metastases for papillary thyroid carcinomas, suggesting that 




Figure 2. Primary medullary thyroid carcinoma. In peritumoral thyroid tissue adjacent to primary tumor (T), small to medium-sized slender to round lymphatic vessels were present as well as few slender small lymphatic vessels at the peripheral tumor zone (A). There were no lymphatic vessels in midst of the tumor (B). Another area of the same case revealed much more slender lymphatic vessels (I) in normal thyroid tissue adjacent to the tumor extending into the peripheral tumor zone (C). The same case showed larger round venous vessels adjacent to the tumor (B) and there were numerous slender to enlarged round venous vessels at the peripheral tumor zone (D). (A) and (C): LYVE-1, (B) and (D): F-8.

proliferation and invasion of new lymphatic vessels into the tumor play a role in its spread through lymphatic vessels [4]. Follicular thyroid carcinomas metastasize to the distant organs through hematogenous spread rather than through lymph spread [8]. Thus, MTCs have a propensity of both papillary and follicular thyroid carcinomas in regards to the tumor spreading patterns. This study aimed to investigate both lymphatic and venous vessels in the peri- and intratumoral vessels by immunocytochemical staining for lymphatic vessels using LYVE-1 and venous vessels using F-8 immunocytochemical staining.

Peritumoral thyroid tissues revealed moderately increased lymphatic vessels and more increased venous vessels at 2.3 times and 3.7 times more lymphatic and venous vessels than the normal thyroid glands, respectively (Table 1). These results support that tumor-induced lymphangiogenesis and angiogenesis was evident at the peritumoral thyroid tissues whereas there were also some but less lymphangiogenesis and angiogeneiss at the peripheral zone of the invading MTC tumors (Figure 2(A) and Figure 2(C)). These increased lymphatic and venous vessels were also evident at the margin of metastatic lymph nodes (Figure 3(C) and Figure 3(D)). In the midst of metstatic lymph nodes, there were no lymphatic vessels but some small venous vessels by F-8 immunostaining (Figures 3(A)-(D)).

There was a considerable difference of lymphatic and venous vessels between metastatic lymph nodes and tumor-free lymph nodes: metastatic lymph nodes revealed slight to moderately increased lymphatic vessels whereas venous vessels were moderately more increased at the invading metastatic margin (Figure 3(C) and Figure 3(D)). Lymphatic vessels in metastatic lymph nodes increased $24 \%$ and venous vessels increased 5.6 times than tumor-free lymph nodes (Table 1). This markedly increased venous vessels in metastatic lymph 


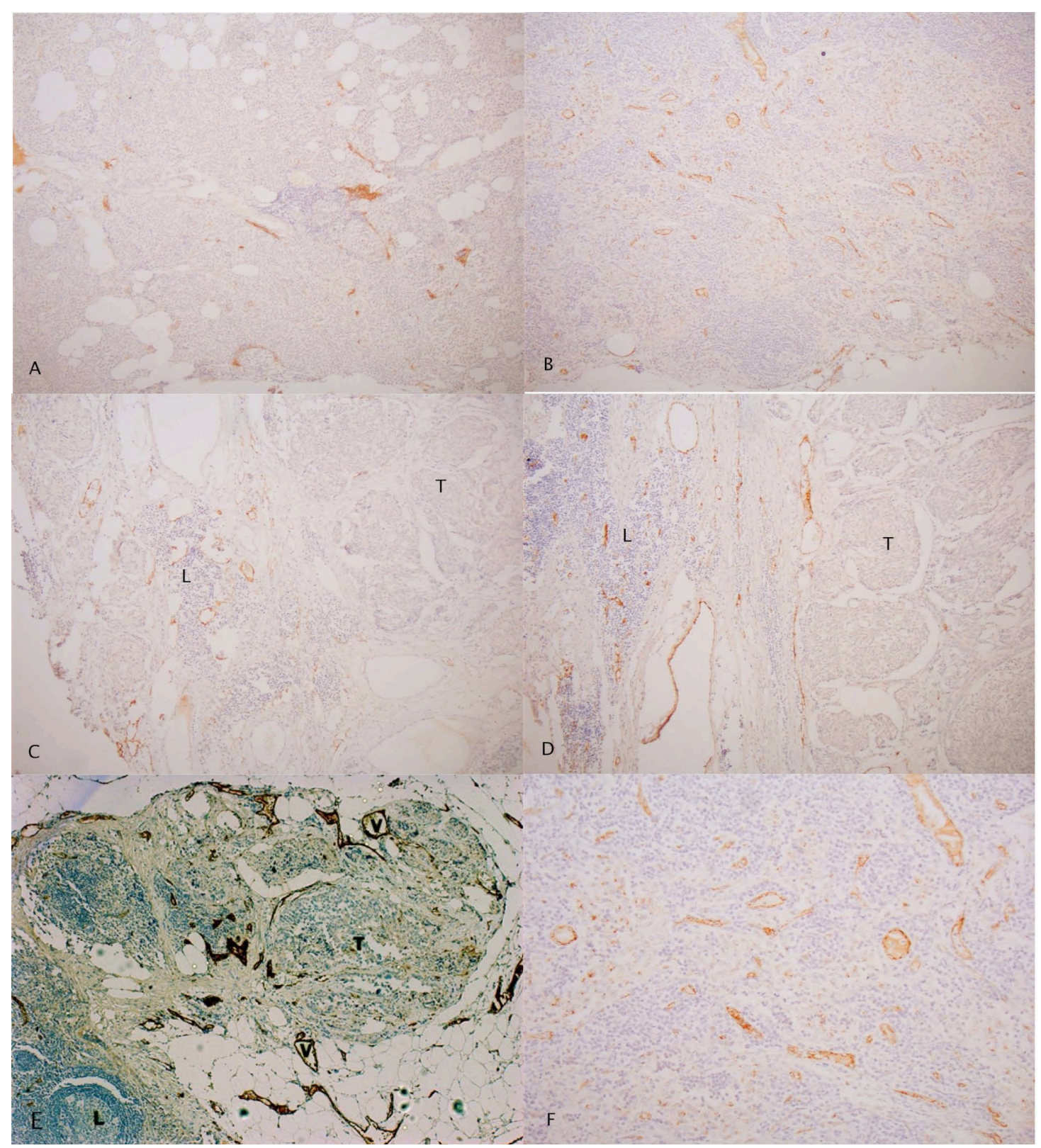

Figure 3. Normal (A) and (B) and metastatic lymph nodes (C) to (E). Normal lymph nodes contained sporadically distributed slender to round lymphatic vessels (A) whereas there were more diffusely distributed slender to more round small venous vessels (B). The capsule of metastatic lymph nodes contained numerous slender lymphatic vessels (C) as contrast to dilated larger venous vessels and a few small venous vessels extending into the peripheral tumor zone (D). At the peripheral zone and perilymph node connective tissues of metastatic lymph nodes, there were numerous slender to round venous vessels $(F)$ as well as scattered smaller venous vessels in the midst of the metastatic lymph nodes (E). L: Lymphoid follicle, T: Tumor, v: venous vessels; (A) and (C): LYVE-1, (B), (D) and (E): F-8.

nodes further support an easier access for venous spread in the metastatic lymph nodes. Compared with the ample presence of lymphovascular invasion in colonic carcinomas [17], there was no lymphovascular invasion or tumor embolus in the current MTC cases.

We used routinely formalin-fixed and paraffin-embedded tissue sections in this study and we also used fresh frozen tissues of normal thyroid for LYVE-1 and F-8 staining, with which to compare LYVE-1 and F-8 immunostaining with formalin-fixed and paraffin-embedded thyroid tissues. With frozen sections, more numerous 
slender lymphatic epithelia were strongly stained within interfollicular fibrous stroma in contrast to the less lymphatic epithelia stained in formalin-fixed and paraffin-embedded sections (Figure 1). It is evident that many LYVE-1 positive sinusoidal epithelia and lymphatic vessels were not immunocytochemically stained in formalin-fixed and paraffin-embedded sections. It is very likely that formalin-fixation and paraffin-embedding masked or damaged the LYVE-1 epitope and/or collapsed lymphatic vessels. Therefore, it is very likely that LYVE-1 and F-8 immunostaining for MTCs was under detected by immunocytochemical staining with formalin-fixation and paraffin-embedding and more LYVE-1 and F-8 immunostaining would have been identified by adequate tissue processing as in frozen sections [13] [14].

In papillary thyroid carcinomas, the presence of intratumoral irregular lymphatic vessels supports that there are newly formed, rather than preexisting lymphatic vessels that have been surrounded and entrapped by the tumor growth [6]. The morphologic appearance of these intratumoral lymphatic vessels resembles that of immature tumor venous vessels observed in other tissues [16]. There were similarly irregular lymphatic vessels in peritumoral tissues in MTCs, which match well with the newly formed, entrapped lymphatic vessels in the advancing margins of the tumor, resembling that of immature tumor blood vessels observed in the advancing margins of colonic carcinomas [17]-[20].

Banerji et al. extensively studied LYVE-1 mRNA levels in many organs, finding abundant mRNA in spleen, lymph node, heart, lung and fetal liver RNA whereas a lesser mRNA in appendix, bone marrow, placenta, muscle and adult liver [9]. There was almost completely absent mRNA in peripheral blood lymphocytes, thymus, brain, kidney and pancreas RNA, reflecting pancreatic islets accounting for only $1 \%-2 \%$ of the total pancreatic tissue despite immunocytochemical presence of LYVE-1 in pancreatic islets [22]. Therefore, mRNA levels of many organs do not exactly match with that of antigen protein levels revealed by immunocytochemical staining for LYVE-1 in formalin-fixed and paraffin-embedded tissue sections.

At present, there are no absolutely specific markers for lymphatic vessels and some of the other available markers for lymphatic vessels are: 1) D2-40, a novel monoclonal antibody to 0-linked sialoglycoprotein that reacts with a fixation-resistant epitope in lymphatic endothelium [23]-[25], which was originally produced against a cultured human ovarian adenocarcinoma cell line, HEY [21] and was initially found to immunostain human fetal germ cells, seminomas and dysgerminomas [25] [26]; 2) Podoplanin, a 43 kDA glycoprotein cloned as a cell surface protein expressed on glomelular podocytes [27] [28]; 3) Prox-1, a homeobox gene expressed in a subpolulation of endothelial cells that gives rise to the lymphatic system, which is required for lymphatic development [29].

Thus, the lymphatic vessel comprises a complex network system as characterized as above, and further studies are warranted to clarify and characterize this complex lymphatic vessel system in the endocrine organs and endocrine tumors.

\section{Acknowledgements}

I want to express my sincere thanks to Dr. Ov Slayden for allowing me to use his research laboratory to perform immunocytochemical staining at Reproductive Division, Oregon National Primate Center, Beaverton, OR. This study was supported in part by ONPRC Core Grant: NIH RR 000163.

\section{References}

[1] Messina, M. and Robinson, B.G. (2007) Technology Insight: Gene Therapy and Its Potential Role in the Treatment of Medullary Thyroid Carcinoma. Nature Clinical Practice Nephrology, 3, 290-301.

[2] Rosai, J. (2004) Thyroid Gland. In: Rosai, J., Ed., Ackerman’s Surgical Pathology, 8th Edition, St. Louis, Mosby, 493567.

[3] Marchens, A. and Dralle, H. (2013) Prognostic Impact of N Staging in 715 Medullary Thyroid Cancer Patients: Proposal for a Revised Staging System. Annals of Surgery, 257, 323-329. http://dx.doi.org/10.1097/SLA.0b013e318268301d

[4] Alvizaki, M., Saltiki, K., Rentiziou, G., Papathoma, A., Sariak, L., et al. (2012) Medullary Thyroid Carcinoma: The Influence of Policy Changing in Clinical Characteristics and Disease Progression. European Journal of Endocrinology, 167, 799-808. http://dx.doi.org/10.1530/EJE-12-0388

[5] Balock, Z.W. and LiVolsi, V.A. (2002) Medullary Thyroid Carcinoma. In: LiVolsi, V.A. and Asa, S.L., Eds., Endocrine Pathology, Churchill Livingston, 81-83. 
[6] Hall, F.T., Freeman, J.L., Asa, S.L., Jackson, D.G. and Beasley, N.J. (2003) Intratumoral Lymphatics and Lymph Node Metastases in Papillary Thyroid Carcinoma. Archives of Otolaryngology—Head and Neck Surgery, 129, 716-719. http://dx.doi.org/10.1001/archotol.129.7.716

[7] Giogadze, T.A., Baloch, Z.W., Pasha, T., Zhang, P.J. and LiVolsi, V.A. (2005) Lymphatic and Blood Vessel Density in the Follicular Lesions of Thyroid. Modern Pathology, 18, 1424-1431. http://dx.doi.org/10.1038/modpathol.3800452

[8] Lin, X., Lie, Y. and Silverman, J.F. (2010) Follicular Thyroid Carcinoma Invades Venous Rather than Lymphatic Vessels. Diagnostic Pathology, 5, 1-8. http://dx.doi.org/10.1186/1746-1596-5-8

[9] Banerji, S., Ni, J., Wang, S.W., Clasper, S., Su, J., Thommi, R., Jones, M. and Jackson, D.G. (1999) LYVE-1, a New Homologue of the CD 44 Glycoprotein, Is a Lymph-Specific Receptor for Hyaluronan. The Journal of Cell Biology, 1444, 789-801. http://dx.doi.org/10.1083/jcb.144.4.789

[10] Jackson, D.G., Prevo, R., Clasper, S. and Banerji, S. (2001) LYVE-1, Lymphatic System and Tumor Lymphangiogenesis. Trends in Immunology, 22, 317-321. http://dx.doi.org/10.1016/S1471-4906(01)01936-6

[11] Prevo, R., Banerji, S., Ferguson, J.P., Clasper, S. and Jackson, D.G. (2001) Mouse LYVE-1 Is an Endocytic Receptor for Hyaluronan in Lymphatic Endothelium. Journal of Biological Chemistry, 276, 19420-19430. http://dx.doi.org/10.1074/jbc.M011004200

[12] Jackson, D.G. (2003) The Lymphatics Revisited. New Perspectives from the Hyaluronan Receptor LYVE-1. Trends in Cardiovascular Medicine, 13, 107. http://dx.doi.org/10.1016/S1050-1738(02)00189-5

[13] Tomitat, T. (2008) Immunocytochemical Localization of Lymphatic and Venous Vessels in Colonic Polyps and Adenomas. Digestive Diseases and Sciences, 53, 1880-1885. http://dx.doi.org/10.1007/s10620-007-0078-9

[14] Tomita, T. (2009) LYVE-1 Immunocytochemical Staining for Gastrointestinal Carcinoids. Pathology, 41, $248-253$. http://dx.doi.org/10.1080/00313020902756253

[15] Tomita, T. (1997) Matrix Metalloproteinases and Inhibitors of Matrix Metalloproteinases in Thyroid C-Cells and Medullary Thyroid Carcinomas. Histopathology, 31, 150-156. http://dx.doi.org/10.1046/j.1365-2559.1997.2160834.x

[16] Tomita, T. (2012) Islet Amyloid Polypeptide in Pancreatic Islets from Type 2 Diabetic Subjects. Islets, 4, $223-232$. http://dx.doi.org/10.4161/isl.20477

[17] Kaio, E., Tanaka, S., Kitadai, Y., et al. (2003) Clinical Significance of Aniogenic Factor Expression at the Deepest Invasive Site of Advanced Colorectal Carcinoma. Oncoloty, 64, 61-73. http://dx.doi.org/10.1159/000066511

[18] Petterspn, A., Nagly, A., Brown, L.F., et al. (2000) Heterogeneity of the Angiogenic Response Induced in Different Normal Adult Tissues by Vascular Permeability Factor/Vascular Endothelial Growth Factor. Laboratory Investigation, 80, 99-115. http://dx.doi.org/10.1038/labinvest.3780013

[19] Sharinen, P., Tammela, T., Karkkanen, M.J. and Alitalo, K. (2004) Lymphatic Vasculature: Development, Molecular Regulation and Role in Tumor Metastasis and Inflammation. Trends in Immunology, 25, 387-395. http://dx.doi.org/10.1016/j.it.2004.05.003

[20] Kaneko, I., Tanaka, S., Oka, S., Kawamujra, T., et al. (2007) Lymphatic Vessels Density at the Site of Deepest Penetration of Lymph Node Metastasis in Submucosal Colorectal Carcinoma. Diseases of the Colon \& Rectum, 50, 13-21. http://dx.doi.org/10.1007/s10350-006-0745-5

[21] Sipos, B., Klapper, W., Kruse, M.L., Kaltoff, H., et al. (1999) Expression of Lymphangiogenesis Factors and Evidences of Intratumoral Lymphangiogenes in Pancreatic Endocrine Tumors. American Journal of Pathology, 154, 385394.

[22] Tomita, T. (2007) Lymphatic Vessel Endothelial Hyaluronan Receptor-1 Immunocytochemical Staining for Pancreatic Islets and Pancreatic Endocrine Tumors. Pancreas, 35, e18-e22. http://dx.doi.org/10.1097/MPA.0b013e318068fcba

[23] Kahn, H.J. and Marks, A. (2002) A Monoclonal Antibody, D2-40, for Detection of Lymphatic Invasion in Primary Tumors. Laboratory Investigation, 82, 1255-1257. http://dx.doi.org/10.1097/01.LAB.0000028824.03032.AB

[24] Fukunaga, M. (2005) Expression of D2-40 in Lymphatic Endothelium of Normal Tissues and Vascular Tumours. Histopathology, 46, 396-402. http://dx.doi.org/10.1111/j.1365-2559.2005.02098.x

[25] Franke, F.E., Pauls, K., Rey, R., Marks, A., Bergmann, M. and Steger, K. (2004) Differentiation Markers of Sertoli Cells and Germ Cells in Fetal and Early Postnatal Human Testis. Anatomy and Embryology (Berlin), 209, 169-177.

[26] Bailey, D., Baumel, R., Law, J., Shelidan, K. and Kahn, H.J. (1986) Production of Monoclonal Antibody Specific for Seminomas and Dystgerminomas. Proceedings of the National Academy of Sciences of the United States of America, 83, 5291-5295. http://dx.doi.org/10.1073/pnas.83.14.5291

[27] Breitender-Geleff, S., Soleiman, A., Kawalski, H., et al. (1999) Angiosarcomas Express Mixed Endothelial Phenotypes of Blood and Lymphatic Capillaries: Population as a Specific Marker for Lymphatic Endothelium. American Journal of Pathology, 154, 385-394. http://dx.doi.org/10.1016/S0002-9440(10)65285-6

[28] Adachi, Y., Nakamura, H., Kitamura, Y., Taniguchi, Y., Araki, K., et al. (2007) Lymphatic Vessel Density in Pulmonary 
Adenocarcinoma Immunohistochemically Evaluated with Anti-Podoplanin or Anti-D2-40 Is Correlated with Lymphatic Invasion or Lymph Node Metastasis. Pathology International, 57, 171-177.

http://dx.doi.org/10.1111/j.1440-1827.2007.02077.x

[29] Wigle, J.T. (1999) Prox-1 Function Is Required for the Development of the Murine Lymphatic System. Cell, 98, 769778. http://dx.doi.org/10.1016/S0092-8674(00)81511-1 